Revisión de Lit. Septiembre-Diciembre 2017; 7(3):14-20. Recibido: 15/02/2017 Aceptado: 10/07/2017.

http://dx.doi.org/10.21929/abavet2017.73.1

\title{
Bacillus subtilis como probiótico en avicultura: aspectos relevantes en investigaciones recientes
}

Bacillus subtilis as a probiotic in poultry farming: relevant aspects in recent research

\author{
Medina-Saavedra Tarsicio ${ }^{1 *}$ tarsicioms@hotmail.com, Arroyo-Figueroa \\ Gabriela1 gabiaf@yahoo.com.mx, Herrera-Méndez Carlos ${ }^{1}$ caherhe_23@hotmail.com, \\ Mexicano- Santoyo Lilia² lilia_lasalle@hotmail.com
}

${ }^{1}$ Departamento de Ingeniería Agroindustrial. Campus Celaya Salvatierra. Universidad de Guanajuato. México. ${ }^{2}$ Instituto Tecnológico de Tepic. Tepic Nayarit. *Autor responsable de correspondencia: Arteaga sin número C. P. 38900 Salvatierra, Guanajuato. México.

\section{RESUMEN}

El presente artículo es una recopilación de trabajos donde se observa el uso de los probióticos como alternativa al uso de antibióticos como promotores del crecimiento. Se revisaron las bondades del Basillus subtillis y su uso como probiótico, encontrado en una gran variedad de ambientes. Se ha mostrado que el $B$. subtilis como probiótico es seguro para usar en la alimentación de las aves sin efectos negativos en el medio ambiente. Otra de la característica relevante del $B$. subtilis es su acción en la estabilidad de la microbiaota intestinal al disminuir la presencia de E. Coli, Salmonelas y coccidias, favoreciendo el incremento de microorganismos benéficos y la inmunidad mediante el incremento de $\lg A$ e lgG. En investigaciones recientes se ha encontrado que el $B$. subtilis contribuye en la reducción de niveles de amoniaco en excretas, producción de sustancias antioxidantes y el aumento de la digestibilidad como consecuencia del equilibrio de la ecología intestinal de las aves. Además, también se ha encontrado que las xilanasas que producen los $B$. subtilis tienen un efecto similar a los antibióticos en intestino delgado. Conocer al $B$. subtilis como probiótico representa mejorar parámetros productivos y condiciones sanitarias de las aves.

Palabras clave: antibióticos, inmunidad, productividad, enzimas.

\begin{abstract}
The present article is a compilation of papers that show the use of probiotics as an alternative to the use of antibiotics as growth promoters. The benefits of Basillus subtilis had been found in a wide variety of environments and their use as a probiotic has been extensively studied. It has been shown that $B$. subtilis as a probiotic is of safe use in the feeding broiler without negative effects on the environment. Others of the relevant characteristics of $B$. subtilis are their action on the stability of the intestinal microbial by reducing the presence of $\mathrm{E}$. coli, Salmonella, and Coccidia, favoring the increase of beneficial microorganisms and improving immunity by increasing IgA and IgG. Recent research has shown that $B$. subtilis contributes to the reduction of levels of ammonia in excreta, the production of antioxidant substances and the increase of digestibility because of the equilibrium of the intestinal ecology of birds, it has also been found that xylanases producing $B$. subtilis have a similar effect to antibiotics in the small intestine. Knowing the $B$, subtilis as probiotic represents to improve productive parameters and sanitary conditions of the birds.
\end{abstract}

Keywords: antibiotics, immunity, productivity, enzymes. 


\section{INTRODUCCIÓN}

Los problemas relacionados con los antibióticos, patógenos resistentes y residuos en productos de origen animal, ha limitado el uso de productos sintéticos como promotores de crecimiento en la industria de la producción animal, por lo tanto, se buscan alternativas naturales y seguras. En este sentido, los probióticos, prebióticos y los ácidos orgánicos se han sugerido como los reemplazos más importantes para los antibióticos.

El término "probiótico" se ha usado desde 1965 para describir a un organismo que interviene en el balance de la microflora intestinal; sin embargo, en los últimos años se ha considerado como un suplemento alimenticio microbiano vivo, y los alimentos que los contienen se consideran funcionales (Gibson y Roberfroid, 1995). Las bacterias y levaduras se han utilizado como fuentes benéficas para mantener una microbiota digestiva sana y equilibrada. Para Grethel et. al., (2008) los microorganismos más usados son Lactobacillus sp., Streptococcus faeccium, Bacillus subtilis, Bacillus licheniformis, Bacillus stearothermophyllus y Saccharomyces cerevisiae. Sin embargo, el género Bacilus destacan como probiótico, por la acción de las enzimas hidrofílicas extracelulares que actúan sobre polisacáridos, ácidos nucleicos y lípidos; empleando estos como fuentes de carbono, donadores de electrones y productores de antibióticos (Forte et al., 2016; Manafi et al., 2016).

Dentro del género Bacillus, destacan B. cereus, B. licheniformis, B. subtilis y $B$. natto. (Grethel et. al., 2008). En 1941 los árabes usaban B. subtilis para controlar diarreas consumiéndolo a través del excremento fresco de los camellos. Los alemanes verificaron que la ingestión de $B$. subtilis mejoraba los cuadros entéricos (Guevara, 2011). Kuipers et al. (2000) demostraron que los $B$. Subtilis no causan toxicidad en los animales vertebrados, además de contar con una acción bactericida y fúngica bien estudiada al producir entre otros el antibiótico bacitracina del grupo de los polipeptídicos. Además, demostraron que el $B$. subtilis se ha encontrado en ambientes diversos como el suelo y en el sistema digestivo de los rumiantes (Kuipers et al., 2000). Grethel et al. (2008) mostraron que cuando es ingerido por aves su microbiota intestinal fue estabilizada, disminuyen los microorganismos patógenos e incrementa la población de Lactobacillus $s p$.

De manera general, el presente trabajo tiene como objetivo realizar una revisión sobre los aspectos más importantes encontrados en las investigaciones recientes donde el B. subtilis interviene como probiótico, destacando aspectos importantes en sistemas de producción de las aves.

\section{BACILLUS SUBTILIS COMO ADITIVO SEGURO}

Bacillus subtilis C-3102 ha sido utilizado como probiótico desde 1986 para mejorar el rendimiento productivo en pollos de engorda. La Autoridad Europea de Seguridad Alimentaria (EFSA) elaboraron un informe científico sobre la seguridad y eficacia de 
un producto basado en el $B$. subtilis como aditivo para pollos de engorda con un contenido mínimo de $1 \times 10^{7}$, y uno máximo de $5 \times 10^{7} \mathrm{UFC} / \mathrm{kg}$ en dieta completa. $B$. Subtilis; es una especie con evaluación QPS (Qualified Presumption of Safety) por la EFSA por la sensibilidad a antibióticos y la ausencia de potencial toxigénico, lo que es considerado aditivo seguro para aves, para el consumidor y para el medio ambiente (González 2009).

\section{BACILLUS SUBTILIS EN LA REDUCCIÓN DE BACTERIAS NOCIVAS}

En estudios recientes han mostrado que utilizando el $B$. subtilis como probiótico en gallinas ponedoras (Hy-Line Brown) de 28 semanas de edad, puede favorecer la ganancia productiva mejorando de manera efectiva su rendimiento y la calidad a través de la reducción de $E$. coli fecal y la modulación de la microbiota cecal, mediante el uso de $B$. subtilis como probiótico; encontrando que la alimentación en relación con la producción de huevo disminuye de manera importante, mejorando además la resistencia de la cáscara del huevo (Guo, Dong, Liu, \& Tong, 2016).

Por otra parte, Forte et al., (2016) encontraron que mediante el uso de Lactobacillus acidophilus y $B$. subtilis como probióticos, disminuyeron la presencia de $E$. coli, estafilococos y clostridios; al tiempo que se incrementa la presencia de bacterias beneficiosas, tales como Lactobacillus spp. y Bifidobacterium spp. (Forteet al., 2016).

Otros estudios sugieren que los probióticos formadores de esporas favorecen situaciones de crianza, donde la carga bacteriana por colibacilosis es alta. Al respecto Manafi et al., (2016) evaluó el efecto de B. subtilis y Disalicilato metileno bacitracina en el rendimiento y salud de los pollos machos Ross, después de la inoculación intramuscular con E. coli $\left(0,5 \mathrm{~mL}\right.$ de cultivo que contiene $10^{8}$ UFC de E. coli $\left./ \mathrm{mL}\right)$; provocando la disminución de la altura de las vellosidades y un incremento de presencia de las colonias de E. coli, coliformes y Salmonella en partes cecal de los intestinos de los pollos. El uso del Disalicilato metileno bacitracina sólo ayudó a disminuir la presencia de las bacterias patógenas, pero $B$. subtilis además aumentó la digestibilidad de los nutrientes y la altura de las vellosidades intestinales (Manafi, Khalaji, Hedayati, \& Pirany, 2016).

\section{BACILLUS SUBTILIS Y LA CAPACIDAD ANTIOXIDANTE}

En relación a los efectos del probiótico $B$. subtilis como un aditivo en el alimento, se sabe que mejora el crecimiento y la calidad de la carne de pollos de engorda por su capacidad antioxidante, al adicionarlo en el alimento de las aves; por su parte Bai et al., (2016) reportó un incremento de las concentraciones en suero e hígado de sustancias antioxidantes como el glutatión, glutatión reductasa, glutatión peroxidasa y superóxido dismutasa, y un incremento de las concentraciones séricas de $\lg A$ e $\lg G$.

\section{BACILLUS SUBTILIS Y LA CAPACIDAD PRODUCTIVA}

Investigadores como Bai et al., (2016) reportaron una mejora en el aumento de peso y la tasa de conversión alimenticia en machos Arbor Acres de un día de nacidos, cuando 
se suplementaron con Bacillus subtilis mbJ (BSfmbJ) en dietas basales de 2 , 3 Y 4 x $10^{10}$ UFC/kg, sin antibióticos. Por su parte Zhang (2013) coincide en los beneficios de las dietas suplementadas con $B$. subtilis, encontrando mayor ganancia de peso y conversión alimenticia con el uso del probiótico, superando a las dietas donde se les adicionaba un antibiótico.

\section{BACILLUS SUBTILIS Y LOS BENEFICIOS PARA EL MEDIO AMBIENTE}

El amoniaco proveniente del estiércol, está relacionado con la utilización de nutrientes y el ecosistema de la microflora intestinal, lo que representa un problema ambiental; además de afectar la salud de los animales y de los trabajadores. Se ha visto que con la adición de $B$. subtilis en la alimentación, tiende a influir en el ecosistema de la microflora intestinal; capaz de reducir la emisión de amoniaco en aves de corral, mediante la mejora de la actividad de enzimas y la utilización de nitrógeno (Zhang et al., 2014). Por otro lado, Sharma et al., (2016) realizando mediciones mediante espectrometría de masas, encontraron que una dieta con la adición de $B$. subtilis como probiótico y mezclada con yuca/Quillaja, fue eficaz en la reducción de las emisiones de algunas sustancias olorosas del estiércol de pollo de engorda.

\section{BACILLUS SUBTILIS EN EL ESTIÍMULO DE LA INMUNIDAD}

Se han estudiado las implicaciones que tiene el uso del $B$. subtilis en el desarrollo de la inmunidad de los pollos de engorda, mostrado los efectos inmunomoduladores de $B$. subtilis en la inmunidad innata en pollos de engorda, encontrando niveles elevados de óxido nitroso (ON); el cual actúa como un mensajero intercelular que regula el tono vascular, la activación de las plaquetas y las respuestas inmunes como neurotransmisor en el sistema nervioso central (Pozo et al.,1998; Lee et al., 2011); es una molécula citotóxica implicada en la eliminación de bacterias, virus y protozoos, así como de células tumorales.

Para Lee et. al. (2011) los macrófagos de pollos representaron una fuerte de fagocitosis de Salmonella, mediante mecanismos de autorregulación en la producción del ON; son importantes para mantener la homeostasis, a pesar de los diversos mecanismos microbicidas dependientes o no del oxígeno. De la misma forma que en macrófagos activados durante la respuesta inmune in vivo o expuestos a la acción de citosinas in vitro, aumenta la producción del ON (Gorocica et. al., 1999).

En otros estudios se ha encontrado que una dieta con $B$. subtilis reduce los signos clínicos de la infección por Eimeria maxima en pollos de engorda, y concomitantemente mejora la inmunidad innata y adquirida de una manera dependiente de la cepa bacteriana. Adicionalmente, se encontró que existen otros mecanismos, además de la presencia de $\mathrm{ON}$, que pueden estar involucrados en la mediación de la inmunidad protectora contra Eimeria spp. Además, se han comprobado las propiedades inmunomoduladoras de $B$. subtilis en la coccidiosis aviar, al reducir los signos clínicos de la enfermedad; conduciendo a la mejora de la 
respuesta inmunitaria protectora y/o la promoción de la biosíntesis de péptidos antimicrobianos endógenos (Kyung-Woo et. al.,2010).

\section{BACILLUS SUBTILIS Y LAS FUENTES DE OMEGA 3}

El adecuado crecimiento y desarrollo de los pollos de engorda depende de una apropiada alimentación y la adición de productos nutracéuticos e higiénicamente seguros. El uso de $B$. subtilis en la dieta de los pollos de engorda reduce las concentraciones de colesterol sérico en aves, además, de los ácidos grasos insaturados; sin embargo, la adición de una fuente de omega 3 (Salvia hispanica L.) no produjo una mejora significativa (Fernández et. al.,2014). Por otro lado se ha comprobado que el enriquecimiento de dietas con omega 3 podría disminuir la respuesta inflamatoria, incrementar la eritropoyesis y mejorar la inmunidad y el crecimiento animal (Newman et al., 2002; Saleh et al., 2009).

\section{BACILLUS SUBTILIS Y LA PRODUCCIÓN DE ENZIMAS}

La industria de los alimentos balanceados frecuentemente busca mejorar la energía metabolizable (EM) de los ingredientes de las dietas, una forma de hacerlo es a través de la suplementación con enzimas de tipo polisacaridasas, para mejorar la digestibilidad y elevar su valor nutritivo. La xilanasa es una de las enzimas más utilizadas como producto de la fermentación microbiana. Investigaciones recientes han encontrado que la bacteria $B$ subtilis produce la xilanasa E1606 (Vandeplas \& Bodin, 2012). También se ha visto que las xilanasas tienen un efecto similar a los antibióticos sobre la microflora a nivel del intestino delgado. La reducción de la viscosidad acelera la velocidad del tránsito intestinal y deja menos tiempo a las bacterias para implantarse. Mediante la hidrolización de los arabinoxilanos $(A X)$, las xilanasas permiten reducir el efecto antinutriente y aumentar así el valor alimenticio, liberando los nutrientes contenidos y reduciendo la viscosidad intestinal; debido a que las xilanasas de origen bacteriano reducen la competitividad animal bacterial para con los nutrientes (Vandeplas \& Bodin, 2012).

\section{CONCLUSIONES}

Los probióticos juegan un papel protagónico en la tarea de mantener la microbiología intestinal en un equilibrio adecuado, para garantizar una mejora de la producción y su calidad. Las funciones estudiadas de la bacteria Bacillus subtiles representan el claro ejemplo de lo que es un probiótico; iniciando con la mejora del ecosistema intestinal del ave, mediante una rápida colonización de tracto intestinal para desplazar a los microoganismos patógenos, y así favorecer el desarrollo de otras bacterias benéficas productoras de ácido láctico. La producción de enzimas hidrolíticas favorece la digestión de los alimentos, y el estímulo de la producción de inmunoglobulinas mejora la salud de las aves y como consecuencia se obtiene un mejor rendimiento productivo. 


\section{LITERATURA CITADA}

GONZÁLEZ GB. 2009. Bacillus subtilis se considera seguro para pollos de engorde, para el consumidor y para el medio ambiente. Recuperado el 05 de febrero de 2017, de Albéitar Portal Veterinaria: http://albeitar.portalveterinaria.com/noticia/6945/actualidad/ bacillus-subtilis-seconsidera-seguro-para-pollos-de-engorde-para-el-consumidor-y-para-el-medioambiente.html

BAI K, Huang Q, Zhang J, Fields G, Zhang L, Wang T. 2016. Supplemental effects of probiotic Bacillus subtilis fmbJ on growth performance, antioxidant capacity, and meat quality of broiler chickens. Poultry Science; 96 (1): 74-82. doi: 10.3382 / ps / pew246.

FERNÁNDEZ H, Morales M, Amela M, Salerno C, Rodríguez GH, Arenaz F, Zamponi A. 2014. Efectos de la adición de probiótico (Bacillus subtilis) y omega 3 (Salvia hispanica L.) sobre los parámetros sanguíneos en pollos parrilleros. Rev. Agron. Noroeste Argent. 34 (2): 113-116. ISSN 2314-369X

FORTE C, Acuti G, Manuali E, Casagrande PP, Pavone S, Trabalza MM, Franciosini, M. 2016. Effects of two different probiotics on microflora, morphology, and morphometry of gut in organic laying hens. Poultry Science. 95 (11):2528-2535. DOI: 10.3382 / ps / pew164

GIBSON G, Roberfroid M. 1995. Dietary modulation of the human colonic microbiota: introducing the concept of prebiotics. Journal Nutriology. 125 (6): 1401-1412.

GOROCICA RP, Chávez SR, Lascurain LR, Espinosa MB, Zenteno, GE. 1999. Óxido nítrico, una molécula multifuncional. Rev Inst Nal Enf Resp Mex. 12 (4): 300-304. http://www.medigraphic.com/pdfs/iner/in-1999/in994i.pdf

GRETHEL M, Pérez, M, Bocourt, R. 2008. Empleo de probióticos basado en Bacillus sp y de sus endosporas en la producción avícola. Revista Cubana de Ciencia Agrícola 42, (2): 117-122. ISSN: 0034-7485

GUEVARA J. 2011. Probióticos en nutrición animal. Sistema de Revisiones en Investigación (SIRIVS); 1-10.

veterinaria.unmsm.edu.pe/files/Articulo_guevara_probioticos.pdf

GUO J, Dong X, Liu S, Tong J. 2016. Effects of long-term Bacillus subtilis CGMCC 1.921 supplementation on performance, egg quality, and fecal and cecal microbiota of laying hens. Poultry Science. pew389. doi: 10.3382 / ps / pew389.

KUIPERS O, Konigs W, Kok, J. 2000. Lactic acid bacteria: the bug of the new millennium. Curr Opin Microbiol; 3: 276-282. PMID: 10851157 
KYUNG-WOO L, Hyun, SL, Seung J, Guangxing L, Sung-Hyen L, Erik P, Lillehoj P, Gregory R. 2010. Effect of Bacillus-based direct-fed microbials on Eimeria maxima infection in broiler chickens. Comparative Immunology, Microbiology and Infectious Diseases. 33(6):105-110. DOI:10.1016 / j.cimid.2010.06.001

LEE K, Li G, Lillehoj H, Jang S, Lee S, Babu U, Siragusa G. 2011. Bacillus subtilisbased direct-fed microbials augment macrophage function in broiler chickens. Research in Veterinary Science.91(3):87-91 http://dx.doi.org/10.1016/j.rvsc.2011.01.018.

MANAFI M, Khalaji S, Hedayati M, Pirany N. 2016. Efficacy of Bacillus subtilis and bacitracin methylene disalicylate on growth performance, digestibility, blood metabolites, immunity, and intestinal microbiota after intramuscular inoculation with Escherichia coli in broilers. Poultry Science. 2016 oct 6 . doi: 10.3382 / ps / pew347.

NEWMAN R, Bryden W, Fleck, E, Ashes J, Buttermer W, Storlien L, Downing J. 2002. Dietary $\mathrm{n}-3$ and $\mathrm{n}-6$ fatty acids alter avian metabolism: metabolism and abdominal fat deposition. British Journal of Nutrition. 88:11-18. DOI:10.1079 / BJNBJN2002580

POZO D, Bitzer QO, Osuna C, García PA, Calv JM, Ortíz, GG, Guerrero JM. 1998. Producción de óxido nítrico y su modulación en el sistema inmune y el sistema nervioso. Arch Neurocien Mex. 3(2):84-94.

http://www.imbiomed.com.mx/1/1/articulos.php?method=showDetail\&id_articulo=455 2\&id_seccion=21\&id_ejemplar $=508 \&$ id_revista $=5$

SALEH H. Rahimi S. Karimi TM. 2009. The effect of diet that contained fish oil on performance, serum parameters, the immune system and the fatty acid composition of meat in broilers. International Journal of Veterinary Research; 3(2):69-75. https://ijvm.ut.ac.ir/pdf_20629_72a27f818938aeabcd81dc592662e03e.html

SHARMA NK, Choct M, Dunlop MW, Wu SB., Castada HZ, \& Swick, R. A. 2016. Characterisation and quantification of changes in odorants from litter headspace of meat chickens fed diets varying in protein levels and additives. Poultry Science, 96(4):851-860. DOI: 10.3382 / PS / PEW309.

VANDEPLAS S, Bodin JC. 2012. Acción de una xilanasa producida por Basilus subtilis. Efectos sobre la flora intestinal y el estado sanitario de las aves. Selecciones Avícolas, noviembre. Noviembre 2012: 19-22. ISSN 0210-0541

ZHANG ZF, Cho JH, Kim I. 2013. Effects of Bacillus subtilis UBT-MO2 on growth performance, immune organ relative weight, fecal gas concentration and intestinal microbial shedding in broiler chickens. Livest. Sci. 155:343 - 347. DOI: 10.1016 / j.livsci.2013.05.021. 